\title{
Existence and properties of a value allocation for an economy with differential information
}

\author{
Stefan Krasa, Nicholas C. Yannelis * \\ Department of Economics, University of Illinois at Urbana-Champaign, Box 111, \\ 330 Commerce West Building, 1206 South Sixth Street, Champaign, IL 61820, USA
}

Submitted September 1994; accepted February 1995

\begin{abstract}
We prove the existence of a private value allocation for an economy with differential information where the commodity space may be infinite dimensional, and there is a continuum of states. We also discuss the existence, non-existence, and properties of two alternative value allocation concepts.
\end{abstract}

JEL classification: D8; D82; C7; C71; C72

Keywords: Private value allocation; Differential information economies; Coarse and fine value allucation

\section{Introduction}

In a companion paper, Krasa and Yannelis (1994) introduced the concept of a private value allocation for an economy with differential information. This concept was presented as an alternative to the rational expectation equilibrium notion. In particular, we demonstrated that the private value allocation is coalitionally incentive-compatible and it takes into account the informational advantage or superiority of an individual. Moreover, we showed by means of examples that the private value allocation yields sensible and reasonable outcomes in situations where any rational expectation equilibrium notion fails to do so.

\footnotetext{
* Corresponding author.
} 
In view of the fact that the private value allocation seems to be a successful alternative to the rational expectation equilibrium, it is important to know the conditions that guarantee its existence. Although in Krasa and Yannelis it was mentioned that the private value allocation may exist under fairly mild assumptions, no existence proof was provided. It is the main purpose of the present paper to present sufficient conditions for the existence of a private value allocation. Moreover, we examine the existence and interpretation of two alternative value allocation concepts, i.e. the coarse and the fine value allocations.

It should be noted that for the deterministic value allocation (either ordinal or cardinal value) several general existence results are available in the literature, e.g. Shapley (1969), Shafer (1980), Yannelis (1983), Emmons and Scafuri (1985). However, none of these results can be applied to differential information economies directly. In particular, the presence of a continuum of states, even with a finite-dimensional commodity space, necessitates the use of functional-analytic and measure-theoretic methods.

The paper proceeds as follows. In Section 2 the economy with differential information is introduced. Section 3 defines the private value allocation. Section 4 is focused on the existence proof, and finally Section 5 discusses the coarse and the fine value allocations.

\section{The model}

Let $Y$ denote the commodity space. In our existence result in Section 4, $Y$ may be infinite dimensional. Hence, infinitely many commodities are permissible. ${ }^{1} \mathrm{We}$ consider an exchange economy that extends over two time periods, $t=0$, 1 , where consumption takes place in $t=1$. At $t=0$ there is uncertainty over the state of nature described by a complete probability space $(\Omega, \mathscr{F}, \mu)$. Let $I=\{1, \ldots, n\}$ denote the set of all agents. In $t=0$ agents will agree on net-trades that may be contingent on the state of nature in $t=1$. However, agents are differentially informed with respect to the true state of nature. Specifically, we assume that at $t=1$ agents do not necessarily know which state $\omega \in \Omega$ has actually occurred. They know their own endowment realization and every agent $i$ might have some additional information about the state described by a $\sigma$-algebra $\mathscr{F}_{i}$ with $\mathscr{F}_{i} \subset \mathscr{F}$. Although all our results will be proved for arbitrary information $\sigma$-algebras, it is easier to understand the interpretation of the information $\sigma$-algebras by considering partitions. Thus, assume, for example, that $\mathscr{F}_{i}$ is generated by a countable partition $A_{k}, k \in \mathbb{N}$. Let $\bar{\omega}$ be the true state of the economy in $t=1$. Then agent $i$ observes the event $A_{k}$, which contains $\bar{\omega}$. However, he/she does not know which state $\omega \in A_{k}$ has actually occurred.

\footnotetext{
${ }^{1} Y$ can be any Banach lattice with an order-continuous norm. $Y_{+}$denotes the positive cone of $Y$ (see Section 4 for the appropriate definitions).
} 
By assumption, agents can always observe their own endowment realization. Thus, we can assume without loss of generality that agent $i$ 's initial endowment, $e_{i}$, is measurable with respect to $\mathscr{F}_{i}$

In summary, an exchange economy with differential information is given by $\mathscr{E}=\left\{\left(X_{i}, u_{i}, e_{i}, \mathscr{F}_{i}, \mu\right): i=1, \ldots, n\right\}$ where

(1) $X_{i}: \Omega \rightarrow 2^{Y_{+}}$is the consumption set of agent $i$,

(2) $u_{i}: Y_{+} \rightarrow \mathbb{R}_{+}$is the utility function of agent $i,{ }^{2}$

(3) $\mathscr{F}_{i}$ is a $\sigma$-algebra with $\mathscr{F}_{i} \subset \mathscr{F}$ denoting the private information of agent $i$,

(4) $e_{i}: \Omega \rightarrow Y_{+}$is the initial endowment of agent $i$, where each $e_{i}$ is $\mathscr{F}_{i}$-measurable, (Bochner) integrable ${ }^{3}$ and $e_{i}(\omega) \in X_{i}(\omega) \mu$-a.e.,

(5) $\mu$ is a probability measure on $\Omega$ denoting the common prior of each agent. The expected utility of agent $i$ is given by

$$
\int_{\Omega} u_{i}\left(x_{i}(\omega)\right) \mathrm{d} \mu(\omega){ }^{4}
$$

Throughout the paper, we assume that the utility function $u_{i}$ of each agent $i$ is (weakly) continuous, concave and bounded.

\section{The private value allocation}

We now describe the notion of a private value allocation. This notion is the analog of the private core of Yannelis (1991) and was introduced in Krasa and Yannelis (1994). The main idea for this concept is that each agent $i$ 's trades are restricted to those that are measurable with respect to their private information. Since the Shapley value measures the marginal contribution of each agent to any coalition of which the agent is a member, the assumption that an agent's trade within the coalition must be measurable with respect to the agent's information implies that information asymmetries matter. Since the main focus of this paper is to provide a general existence result, the interested reader is referred to Krasa and Yannelis (1994) for a further discussion of the properties of the private value allocation.

As in the definition of the standard value allocation concept, we must first derive a transferable utility (TU) game in which each agent's utility is weighted by a factor $\lambda_{i}(i=1,2, \ldots, n)$, which allows interpersonal utility comparisons. In

\footnotetext{
${ }^{2}$ One may also assume that the utility function is random, i.e., $u_{i}$ is a real valued function defined on $\Omega \times Y_{+}$. All the results of the paper will remain valid.

${ }^{3}$ See Section 4 for a definition of the Bochner integral. If $Y_{+}=\mathbb{R}^{n}$ this is the standard Lebesgue integral.

${ }^{4}$ Different priors and updating of priors could be introduced as in Krasa and Yannelis (1994, footnote 7).
} 
the value allocation itself no side-payments are necessary. ${ }^{5} \mathrm{~A}$ game with side-payments is then defined as follows.

Definition 1. A game with side-payments $\Gamma=(I, V)$ consists of a finite set of agents $I=\{1, \ldots, n\}$ and a superadditive, real valued function $V$ defined on $2^{l}$ such that $V(\emptyset)=0$. Each $S \subset I$ is called a coalition and $V(S)$ is the 'worth' of the coalition $S$.

The Shapley value of the game $\Gamma$ (Shapley, 1953) is a rule that assigns to each agent $i$ a 'payoff', $\mathrm{Sh}_{i}$, given by the formula ${ }^{6}$

$$
\operatorname{Sh}_{i}(V)=\sum_{\substack{S \subset I \\ S \supset(i)}} \frac{(|S|-1) !(|I|-|S|) !}{|I| !}[V(S)-V(S \backslash\{i\})] .
$$

The Shapley value has the property that $\sum_{i \in I} \operatorname{Sh}_{i}(V)=V(I)$, i.e. the Shapley value is Pareto efficient.

We now define for each economy with differential information, $\mathscr{E}$, and for each set of weights, $\left\{\lambda_{i}: i=1, \ldots, n\right\}$, the associated game with side-payments $\left(I, V_{\lambda}^{p}\right)$ (we also refer to this as a 'transferable utility' (TU) game) as follows:

For every coalition $S \subset I$ let

$$
V_{\lambda}^{p}(S)=\max _{x_{i}} \sum_{i \in S} \lambda_{i} \int u_{i}\left(x_{i}(\omega)\right) \mathrm{d} \mu(\omega),
$$

subject to

(i) $\sum_{i \in S} x_{i}(\omega)=\sum_{i \in S} e_{i}(\omega), \mu$-a.e.,

(ii) $x_{i}-e_{i}$ is $\mathscr{F}_{i}$-measurable for every $i \in S$.

We are now ready to define the private value allocation.

Definition 2. An allocation $x: \Omega \rightarrow \prod_{i=1}^{n} Y_{i}$ with $x_{i}(\omega) \in X_{i}(\omega) \mu$-a.e. for all $i$ is said to be a private value allocation of the economy with differential information, $\mathscr{E}$, if the following holds:

(i) Each net-trade $x_{i}-e_{i}$ is $\mathscr{F}_{i}$-measurable,

(ii) $\sum_{i=1}^{n} x_{i}(\omega)=\sum_{i=1}^{n} e_{i}(\omega), \mu$-a.e.,

(iii) There exist $\lambda_{i} \geq 0$, for every $i=1, \ldots, n$, which are not all equal to zero, with $\lambda_{i} f u_{i}\left(x_{i}(\omega)\right) \mathrm{d} \mu(\omega)=\mathrm{Sh}_{i}\left(V_{\lambda}^{p}\right)$ for all $i$, where $\operatorname{Sh}_{i}\left(V_{\lambda}^{p}\right)$ is the Shapley value of agent $i$ derived from the game $\left(I, V_{\lambda}^{p}\right)$, defined in (3.1).

Condition (i) requires individual measurability of net-trades, i.e. net trades can only be contingent on each agent's individual information $\mathscr{F}_{i}$. (ii) is the market

\footnotetext{
${ }^{5}$ See Emmons and Scafuri (1985, p. 60) or Shafer (1980, p. 468) for further discussion.

${ }^{6}$ The Shapley value measure is the sum of the expected marginal contributions an agent can make to all the coalitions of which he/she is a member (see Shapley, (1953)).
} 
clearing condition. (iii) says that the expected utility of each agent multiplied with his/her weight $\lambda_{i}$ must be equal to his/her Shapley value derived from the TU game $\left(I, V_{\Lambda}^{p}\right)$.

An immediate consequence of Definition 2 is that $\operatorname{Sh}_{i}\left(V_{\lambda}^{p}\right) \geq \lambda_{i} \int u_{i}\left(e_{i}\right) \mathrm{d} \mu$ for every $i$, i.e. the value allocation is individually rational. This follows immediately from the fact that the game $\left(V_{\lambda}^{p}, I\right)$ is superadditive for all weights $\lambda$. Similarly, efficiency of the Shapley value for games with side-payments immediately implies that the value allocation is constrained Pareto efficient.

We now state our main existence result.

Theorem 1. Let $\mathscr{E}=\left\{\left(X_{i}, u_{i}, e_{i}, \mathscr{F}_{i}, \mu\right): i=1, \ldots, n\right\}$ be a finite exchange economy with differential information satisfying the following assumptions for each agent:

(A1) The commodity space $Y_{+}$is the positive cone of a Banach lattice $Y$ with an order-continuous norm.

(A2) $X_{i}: \Omega \rightarrow 2^{Y_{+}}$is a convex, closed, non-empty valued correspondence.

(A3) $u_{i}: Y \rightarrow \mathbb{R}_{+}$is continuous, bounded and concave.

Then a private value allocation exists in $\mathscr{E}$.

The technical conditions in (A1) are explained in the following section. Note that (A1) is automatically fulfilled for all finite-dimensional spaces. Other basic examples of Banach lattices with an order-continuous norm are the Lebesgue spaces $L^{p}, 1 \leq p<\infty$ of $\mathbb{R}^{n}$ valued functions.

\section{The existence proof}

The goal of this section is to provide a general existence result. In particular, we show that private value allocations exist in a setting where there is an infinite number of commodities and an infinite number of states of nature. Before proving our existence result, we outline some mathematical preliminaries.

\subsection{Mathematical preliminaries}

Let $(\Omega, \mathscr{F}, \mu)$ be a probability spacc, and $X$ be a Banach space. We denote by $L_{X}^{1}(\mu)$ the space of all equivalence classes of $X$-valued Bochner integrable ${ }^{7}$ functions $f: \Omega \rightarrow X$ normed by

$$
\|f\|_{1}=\int\|f(\omega)\| \mathrm{d} \mu(\omega)
$$

\footnotetext{
${ }^{7}$ The Bochner integral of a function $f$ can be obtained by approximating $f$ by a sequence of simple functions $f_{n}$ (for a definition of simple functions see footnote 15). This is the same construction that is used to define the Lebesgue integral for $X=\mathbb{R}^{n}$.
} 
It is a standard result that when normed by the functional $\|\cdot\|_{1}$ above, $L_{X}^{1}(\mu)$ becomes a Banach space (see Diestel and Uhl, 1977, p. 50).

We now collect some basic results on Banach lattices (for an excellent treatment see Aliprantis and Burkinshaw, 1985). Recall that a Banach space $X$ is a Banach lattice if there exists an ordering $\geq$ on $X$ with the following properties:

(i) $x \geq y$ implies $x+z \geq y+z$ for every $z \in X$,

(ii) $x \geq y$ implies $\lambda x \geq \lambda y$ for every scalar $\lambda \geq 0$,

(iii) for all $x, y \in X$ there exists a supremum (denoted by $x \vee y$ ) and an infimum (denoted by $x \wedge y$ ),

(iv) $|x| \geq|y|$ implies $\|x\| \geq\|y\|$ for all $x, y \in X$.

As usual, $x^{+}=x \vee 0, x^{-}=(-x) \vee 0$, and $|x|=x^{+}+x^{-}$. We call $x^{+}$and $x^{-}$ the positive and negative parts of $x$, respectively, and $|x|$ the absolute value of $x$. For $x, y \in X$ we define the order interval $[x, y]$ as follows:

$$
[x, y]=\{z \in X: x \leq z \leq y\} .
$$

Note that $[x, y]$ is convex and norm-closed, hence weakly closed (recall Mazur's Theorem). A Banach lattice $L$ is said to have an order-continuous norm if $x_{\alpha} \downarrow^{8}$ in $L$ implies $\left\|x_{\alpha}\right\| \downarrow 0$. A very useful result, which will play an important role, is that if $X$ is a Banach lattice, then the fact that $X$ has an order-continuous norm is equivalent to the weak compactness of order intervals (see, for example, Aliprantis and Burkinshaw, 1985).

We finally note that Cartwright (1974) has shown that if $X$ is a Banach lattice with an order-continuous norm (or, equivalently, $X$ has weakly compact order intervals), then $L_{X}^{1}(\mu)$ has weakly compact order intervals, as well. Cartwright's Theorem will play a crucial role in our existence proof.

\subsection{Proof of Theorem 1}

Let $\left\{\left(X_{i}, u_{i}, e_{i}\right): i=1,2, \ldots, n\right\}$ be an exchange economy where

(a) $X_{i} \subset \mathbb{R}_{+}^{1}$ is the consumption set of agent $i$,

(b) $u_{i}: X_{i} \rightarrow \mathbb{R}_{+}$is the utility function of agent $i$,

(c) $e_{i} \in X_{i}$ is the initial endowment of agent $i$.

Given an economy, $\left\{\left(X_{i}, u_{i}, e_{i}\right): i=1,2, \ldots, n\right\}$, and a set of weights, $\left\{\lambda_{i}\right.$ : $i=1, \ldots, n\}$, where $\lambda_{i} \geq 0$ for every $i$ and $\sum_{i=1}^{n} \lambda_{i}=1$, define the game

$$
V_{\lambda}(S)=\max _{x_{i} \in X_{i}} \sum_{i \in S} \lambda_{i} u_{i}\left(x_{i}\right) \text {, subject to } \sum_{i \in S} x_{i}=\sum_{i \in S} e_{i} \text {. }
$$

Denote by $\operatorname{Sh}_{i}\left(V_{\lambda}\right)$ the Shapley value of agent $i$. The allocation:

$$
x=\left(x_{1}, \ldots, x_{n}\right) \in \prod_{i=1}^{n} X_{i},
$$

\footnotetext{
${ }^{8} x_{\alpha} \downarrow 0$, means that $x_{\alpha}$ is a decreasing net with $\inf _{\alpha} x_{\alpha}=0$.
} 
is said to be a $\lambda$-transfer value allocation or a cardinal value allocation for the economy $\left\{\left(X_{i}, u_{i}, e_{i}\right) ; i=1, \ldots, n\right\}$ if

(i) $\sum_{i=1}^{n} x_{i}=\sum_{i=1}^{n} e_{i}$, and

(ii) there exists $\left\{\lambda_{i} \geq 0: i=1, \ldots, n\right\}$ with $\sum_{i=1}^{n} \lambda_{i}=1$ such that $\lambda_{i} u_{i}\left(x_{i}\right)=$ $\mathrm{Sh}_{i}\left(V_{\lambda}\right)$ for each $i$.

Emmons and Scafuri (1985) or Shapley (1969) show that if $u_{i}$ is concave, and continuous, and if $X_{i}$ is bounded from below, closed and convex, then a cardinal value allocation exists for the economy $\left\{\left(X_{i}, u_{i}, e_{i}\right): i=1, \ldots, n\right\}$. Let $L_{X_{i}}$, denote the set of all functions $x_{i}: \Omega \rightarrow Y$ that are $\mathscr{F}_{i}$-measurable, Bochner integrable, and for which $x_{i}(\omega) \in X_{i}(\omega)$, $\mu$-a.e. Define $W_{i}: L_{X_{i}} \rightarrow \mathbb{R}$ by $W_{i}(x)=\int u_{i}\left(x_{i}(\omega)\right)$ $\mathrm{d} \mu(\omega)$.

Following Bewley's (1972) argument, we will prove our theorem by considering its trace in finite dimensions and appealing to the Emmons and Scafuri (1985) existence result. We first need to prove some simple facts:

(F1) $L_{X_{i}}$ is non-empty.

(F2) $L_{X_{i}}$ is convex, norm-closed and bounded from below.

(F3) $W_{i}$ is weakly upper semi-continuous on $L_{X_{i}}$

(F4) $W_{i}$ is concave on $L_{X_{i}}$.

Fact (F1) follows immediately. Since, by assumption, $e_{i}$, is $\mathscr{F}_{i}$-measurable and Bochner integrable, we can conclude that $e_{i} \in L_{X_{i}}$. Fact (F2) follows directly from assumption (A2). Fact (F3) is proved in Balder and Yannelis (1993, theorem 2.8) and (F4) follows directly from the concavity of $u_{i}$.

Now consider the economy, $\overline{\mathscr{E}}=\left\{\left(L_{X_{i}}, W_{i}, e_{i}\right): i=1, \ldots, n\right\}$, where $L_{X_{i}}$ denotes the consumption set of agent $i$, where $W_{i}$ is the utility function of agent $i$, and where $e_{i} \in L_{X_{i}}$ denotes the initial endowment of agent $i$. Note that the existence of a cardinal value allocation in $\overline{\mathscr{E}}$ implies the existence of a private value allocation for the original economy $\mathscr{E}$.

Let $\mathscr{A}$ be the set of all finite-dimensional subspaces of $L_{Y}^{1}(\mu)$ containing the initial endowments. For each $\alpha \in \mathscr{A}$, let $L_{X_{i}}^{\alpha}=L_{X_{i}} \cap \alpha$ be the consumption set of agent $i$ and $W_{i}^{\alpha}: L_{X_{i}}^{\alpha} \rightarrow \mathbb{R}$ be the utility function of agent $i$. Note that $W_{i}^{\alpha}$ is continuous, since it is the expected utility over a finite-dimensional space. ${ }^{9}$ For each $\alpha \in \mathscr{A}$, we have an economy $\overline{\mathscr{E}}^{\alpha}$ with a finite-dimensional consumption space. Further, for each $\alpha \in \mathscr{A}$, the economy $\overline{\mathscr{E}}^{\alpha}$ fulfills the assumptions of Emmons and Scafuri (1985). Thus, there exists a cardinal value allocation, i.e. there exist $x^{\alpha} \in \prod_{i=1}^{n} L_{X_{i}}^{\alpha}$ such that

(i) $\sum_{i=1}^{n} x_{i}^{\alpha}=\sum_{i=1}^{n} e_{i}$,

(ii) there exist $\lambda_{i}^{\alpha} \geq 0$ with $\sum_{i=1}^{n} \lambda_{i}^{\alpha}=1$, such that $\lambda_{i}^{\alpha} W_{i}^{\alpha}\left(x_{i}^{\alpha}\right)=S h_{i}\left(V_{\lambda^{\alpha} W^{\alpha}}\right)$

\footnotetext{
${ }^{9}$ This follows automatically from the Lebesgue dominated convergence theorem, since each $u$ is, by assumption, continuous and bounded.
} 
for every $i$, where $\operatorname{Sh}_{i}\left(V_{\lambda^{\alpha} \mathrm{W}^{\alpha}}\right)$ is the Shapley value of agent $i$ derived from the game $\left(I, V_{\lambda^{\alpha} W \alpha}\right) .{ }^{10}$

By (i) we have that

$$
0 \leq \sum_{i=1}^{n} x_{i}^{\alpha}=\sum_{i=1}^{n} e_{i}=e
$$

Hence, each $x_{i}^{\alpha}$ lies in the order interval $[0, e]$ in $\sum_{i=1}^{n} L_{X_{i}} \subset L_{Y}^{1}(\mu)$, which is weakly compact by Cartwright's Theorem (see Cartwright, 1974, or Subsection 4.1).

Order the set $\mathscr{A}$ by inclusion. Then $\left\{\left(x_{1}^{\alpha}, \ldots, x_{n}^{\alpha}, \lambda_{1}^{\alpha}, \ldots, \lambda_{n}^{\alpha}\right): \alpha \in \mathscr{A}\right\}$ is a net in $K=\prod_{i=1}^{n}[0, e] \times \Delta$, where $\Delta$ denotes the $(n-1)$-dimensional simplex. Since $K$ is compact we can therefore assume without loss of generality that the net converges to a point $\left(x_{1}, \ldots, x_{n}, \lambda_{1}, \ldots, \lambda_{n}\right){ }^{11}$ To complete the proof we must show that this limit is a value allocation of our original economy, $\mathscr{E}$; i.e. that conditions (i)-(iii) in Definition 2 hold. (i) and (ii) follow immediately, since, by Mazur's Theorem, $L_{X_{i}}$ is weakly closed and hence contains the limit points of the net $x_{i}^{\alpha}, \alpha \in A$.

We now prove (iii). We first show that $\lim _{\alpha} V_{\lambda^{\alpha} W^{\alpha}}(S)=V_{\lambda W}(S)$. Note that weak upper semi-continuity implies that:

$$
\begin{aligned}
\lim _{\alpha} \sup V_{\lambda^{\alpha} W^{\alpha}}(S) & =\lim _{\alpha} \sup \sum_{i \in S} \lambda_{i}^{\alpha} W_{i}^{\alpha}\left(x_{i}^{\alpha}\right), \\
& =\sum_{i \in S} \lim _{\alpha} \sup \lambda_{i}^{\alpha} W_{i}\left(x_{i}^{\alpha}\right) \\
& \leq \sum_{i \in S} \lambda_{i} W_{i}\left(\lim _{\alpha} x_{i}^{\alpha}\right) \\
& =\sum_{i \in S} \lambda_{i} W_{i}\left(x_{i}\right)=V_{\lambda W}(S) .
\end{aligned}
$$

Hence,

$$
\lim _{\alpha} \sup _{\lambda^{\alpha} W^{\alpha}}(S) \leq V_{\lambda W}(S) \text {. }
$$

Now choose $x_{i}^{*}$ such that $V_{\lambda W}(S)=\sum_{i \in S} \lambda_{i} W_{i}\left(x_{i}^{*}\right) .{ }^{12}$ Then for every $\varepsilon>0$ there exists $\bar{\alpha}$ such that

$$
V_{\lambda W}(S)=\sum_{i \in S} \lambda_{i} W_{i}\left(x_{i}^{*}\right) \leq \sum_{i \in S} \lambda_{i}^{\alpha} W_{i}\left(x_{i}^{*}\right)+\varepsilon,
$$

\footnotetext{
${ }^{10}$ Clearly, the game $\left(I, V_{\lambda^{\alpha} W^{\alpha}}\right)$ is defined as follows. For cvery coalition $S \subset I$ let $V_{\lambda^{\alpha} W^{\alpha}}(S)=\max _{x_{i} \in L_{X_{i}}^{\alpha}} \sum_{i \in S} \lambda_{i}^{\alpha} W_{i}\left(x_{i}\right)$, subject to $\sum_{i \in S} x_{i}=\sum_{i \in S} e_{i}$.

${ }^{11}$ More precisely, there exists a subnet that converges. For simplicity, we again choose $\mathscr{A}$ as the index set.

${ }^{12}$ Note that $x_{i}^{*}$ exists, since each $W_{i}$ is weakly upper semi-continuous, and since the $x_{i}$ in the optimization problem in footnote 5 are restricted to the weakly compact order interval $\left[0, \Sigma_{i \in S} e_{i}\right]$, because of feasibility.
} 
for every $\alpha>\bar{\alpha}$. Now choose $\bar{\beta}>\bar{\alpha}$ such that $\bar{\beta}$ contains the space spanned by $\left\{x_{1}^{*}, \ldots, x_{n}^{*}\right\}$. Then

$$
\sum_{i \in S} \lambda_{i}^{\alpha} W_{i}\left(x_{i}^{*}\right) \leq \max _{x_{i} \in L_{x_{i}} \cap \alpha} \sum_{i \in S} \lambda_{i}^{\alpha} W_{i}\left(x_{i}\right)=V_{\lambda^{\alpha} W^{\alpha}}(S),
$$

for all $\alpha>\bar{\beta}$ since $x_{i}^{*} \in L_{X_{i}} \cap \alpha$. Thus, (4.2) and (4.3) imply

$$
\lim _{\alpha} \inf V_{\lambda^{\alpha} W^{\alpha}}(S) \geq V_{\lambda W}(S) \text {. }
$$

Hence, (4.1) and (4.4.) imply that $\lim _{\alpha} V_{\lambda^{\alpha} W^{\alpha}}(S)=V_{\lambda W}(S)$. It follows from the continuity of the Shapley value that $\lim _{\alpha} \operatorname{Sh}_{i}\left(V_{\lambda^{\alpha} W^{\alpha}}\right)=\operatorname{Sh}_{i}\left(V_{\lambda W}\right)$. By taking the lim sup on both sides of the equation $\lambda_{i}^{\alpha} W_{i}^{\alpha}\left(x_{i}^{\alpha}\right)=\operatorname{Sh}_{i}\left(V_{\lambda^{\alpha} W^{\alpha}}\right)$ and by using the weak upper semi-continuity of $W_{i}$ we can conclude that

$$
\lambda_{i} W_{i}\left(x_{i}\right) \geq \lim _{\alpha} \sup \lambda_{i}^{\alpha} W_{i}\left(x_{i}^{\alpha}\right)=\lim _{\alpha} \operatorname{supSh}_{i}\left(V_{\lambda^{\alpha} W^{\alpha}}\right)=\operatorname{Sh}_{i}\left(V_{\lambda W}\right) .
$$

However, the Pareto efficiency of the Shapley value implies that the equality must hold in (4.5). Thus, condition (iii) for a Shapley value allocation holds. This completes the proof of the theorem.

\section{The coarse and the fine value allocation}

\subsection{Definitions}

In this section, we introduce two alternative notions of a value allocation for an economy with differential information. The difference stems from the measurability restriction on the type of allocations that are allowed. Both notions are the analogs of the coarse and the fine cores of Wilson (1978). We begin by defining these concepts. First, note that for arbitrary $\sigma$-algebras $\mathscr{F}_{i}, i=1, \ldots, n$, common knowledge information is given by $\wedge_{i=1}^{n} \mathscr{F}_{i}$, which is the intersection of all $\sigma$-algebras $\mathscr{F}_{i}, i=1, \ldots, n$. In contrast, $\vee_{i-1}^{n} \mathscr{F}_{i}$, which is the $\sigma$-algebra generated by the union of the $\sigma$-algebras $\mathscr{F}_{i}, i=1, \ldots, n$, is the pooled information.

For each economy with differential information, $\mathscr{E}$, and each set of weights, $\left\{\lambda_{i}: i=1, \ldots, n\right\}$, we associate a game with side-payments $\left(I, V_{\lambda}^{c}\right)$, (we also refer to this as a 'transferable utility' (TU) game) according to the following rule.

For cvery coalition $S \subset I$ let

$$
V_{\lambda}^{c}(S)=\max _{x_{i}} \sum_{i \in S} \lambda_{i} \int u_{i}\left(x_{i}(\omega)\right) \mathrm{d} \mu(\omega),
$$

subject to

(i) $\sum_{i \in S} x_{i}(\omega)=\sum_{i \in S} e_{i}(\omega), \mu$-a.e.,

(ii) $x_{i}-e_{i}$ is $\wedge_{i \in S} \mathscr{F}_{i}$-measurable for every $i \in S$. 
The coarse value allocation can now be defined by replacing condition (i) in Definition 2 by:

Each net-trade $x_{i}-e_{i}$ is $\bigwedge_{i=1}^{n} \mathscr{F}_{i}$-measurable; and by replacing $V^{p}$ by $V^{c}$ in condition (iii).

Thus, in contrast to the private value, we now require that net-trades within a coalition can only be based on the common knowledge information of an agent.

The second concept that we introduce in this section is the fine value. For each economy with differential information, $\mathscr{E}$, and each set of weights, $\left\{\lambda_{i}: i=\right.$ $1, \ldots, \mathrm{n}\}$, we associate a game with side-payments $\left(I, V_{\lambda}^{f}\right)$ according to the rule:

$$
V_{\lambda}^{f}(S)=\max _{x_{i}} \sum_{i \in S} \lambda_{i} \int u_{i}\left(x_{i}(\omega)\right) \mathrm{d} \mu(\omega),
$$

subject to

(i) $\sum_{i \in S} x_{i}(\omega)=\sum_{i \in S} e_{i}(\omega)$, $\mu$-a.e.,

(ii) $x_{i}-e_{i}$ is $\vee_{i \in S} \mathscr{F}_{i}$-measurable for every $i \in S$.

The fine value allocation can now be defined by replacing condition (i) in Definition 2 by:

Each net-trade $x_{i}-e_{i}$ is $V_{i=1}^{n} \mathscr{F}_{i}$-measurable; and by replacing $V^{p}$ by $V^{f}$ in condition (iii).

Thus, in contrast to the private value and the coarse value we now require that net-trades within a coalition can only be based on the pooled information of a coalition.

\subsection{The fine value allocation}

One can easily check that the existence of a fine value allocation follows immediately from the existence result for the private value allocation. However, it does not take information asymmetries into account as the theorem below indicatcs.

Theorem 2. Let $\mathscr{E}$ be a differential information economy for which the conditions of Theorem 1 hold. Let $\mathscr{E}$ denote the economy with complete information, i.e. where we replace each $\mathscr{F}_{i}$ by $\mathscr{F}$. Then for every fine value allocation $x^{i}, i \in I$ of $\mathscr{E}$ there exists a fine value allocation $\tilde{x}^{i}, i \in I$ for the economy $\tilde{\mathscr{E}}$ such that weights $\lambda_{i}$ and the agents' expected utilities in both economies are the same.

In order to prove the theorem we need the following result. 
Lemma 1. Let $\mathscr{G}_{i}$ be the $\sigma$-algebra generated by $e_{i}$ and let $\mathscr{G}=\mathrm{V}_{i \in S} \mathscr{G}_{i}$. Then for every allocation $x^{i}, i \in S$ that is feasible for coalition $S$ there exists an allocation $\tilde{x}^{i}, i \in S$ that is feasible for coalition $S$ such that

$$
\int u_{i}\left(\tilde{x}_{i}(\omega)\right) \mathrm{d} \mu(\omega) \geq \int u_{i}\left(x_{i}(\omega)\right) \mathrm{d} \mu(\omega),
$$

for every $i \in S$ and such that each $\tilde{x}_{i}$ is $\mathscr{G}$-measurable.

Proof. We prove Lemma 1 in two steps. In Step 1 we assume that $g$ can be represented by a partition $A_{k}, k \in \mathbb{N}$ of $\Omega$. In Step 2 we prove the general case.

Step 1. Without loss of generality we can assume that $\mu\left(A_{k}\right)>0$ for every $k \in \mathbb{N}$, since we can modify $x_{i}, i \in S$ arbitrarily on sets of measure 0 . Let $e_{S}=\sum_{i \in S} e_{i}$. Then $e_{S}$ is $\mathscr{G}$-measurable and therefore constant on each set $A_{k}$. For each agent $i \in S$ and for each $k \in \mathbb{N}$ define

$$
c_{i}^{k}=\frac{1}{\mu\left(A_{k}\right)} \int_{A_{k}} x_{i}(\omega) \mathrm{d} \mu(\omega) .
$$

Let $\tilde{\boldsymbol{x}}_{i}=\sum_{k \in \mathbb{N}} c_{i}^{k} \mathbf{1}_{A_{k}}$, where $\mathbf{1}_{A_{k}}$ denotes the characteristic function of the set $A_{k} \cdot{ }^{13}$ Note that Jensen's inequality ${ }^{14}$ and (5.4) implies that

$$
u_{i}\left(c_{i}^{k}\right) \geq \frac{1}{\mu\left(A_{k}\right)} \int_{A_{k}} u_{i}\left(x_{i}^{k}\right) \mathrm{d} \mu(\omega)
$$

Now (5.3) follows by first multiplying both sides of (5.5) with $\mu\left(A_{k}\right)$ and then summing both sides with respect to $k \in \mathbb{N}$.

We must now prove that $x_{i}, i \in S$ is feasible for the coalition $S$. This follows immediately by summing both sides of (5.4) with respect to $i \in S$. Thus, for any $\bar{\omega} \in A_{k}$ we get

$$
\begin{aligned}
\sum_{i \in S} \tilde{x}_{i}(\bar{\omega}) & =\sum_{i \in S} c_{i}^{k} \\
& =\frac{1}{\mu\left(A_{k}\right)} \sum_{i \in S} \int_{A_{k}} x_{i}(\omega) \mathrm{d} \mu(\omega) \\
& =\frac{1}{\mu\left(A_{k}\right)} \int_{A_{k}} \sum_{i \in S} x_{i}(\omega) \mathrm{d} \mu(\omega) \\
& =\frac{1}{\mu\left(A_{k}\right)} \int_{A_{k}} e_{S}(\omega) \mathrm{d} \mu(\omega)=\frac{1}{\mu\left(A_{k}\right)} e_{S}(\bar{\omega}) \mu\left(A_{k}\right)=e_{S}(\bar{\omega}) .
\end{aligned}
$$

\footnotetext{
${ }^{13}$ That is, $\mathbf{1}_{A_{k}}(\omega)=1$ if and only $\omega \in A_{k}$ and $\mathbf{1}_{A_{k}}(\omega)=0$, otherwise.

${ }^{14}$ If $\mathrm{E}$ denotes the expected value and if $u$ is concave and bounded then Jensen's inequality implies $u(\mathrm{E}(f)) \geq \mathrm{E}(u(f))$, for any integrable function $f$. In our case, $\mathrm{E}(f)=\left(1 / \mu\left(A_{k}\right)\right) \int_{A_{k}} f(\omega) \mathrm{d} \mu(\omega)$.
} 
Note that we can take $e_{S}$ out of the integrals since $e_{S}$ is constant on $A_{k}$. Hence $\tilde{x}_{i}$, $i \in S$ is feasible. This concludes the proof of Step 1 .

Step 2. We now use an approximation argument to prove the result for arbitrary $\mathscr{G}$. Note that by the definition of the Bochner integral there exists a sequence of simple functions ${ }^{15} e_{i}^{n}$ such that $e_{i}^{n}$ converges in the norm to $e_{i}$ for each $i \in S$ and for which $e_{i}^{n} \leq e_{i}$. Similarly, we can also find a sequence of functions $x_{i}^{n}, n \in \mathbb{N}$ such that $x_{i}^{n}$ converges in the norm to $x_{i}$ and $\sum_{i \in S} x_{i}^{n}=\sum_{i \in S} e_{i}^{n}$ for each $n \in \mathbb{N}$.

Let $\mathscr{G}_{i}^{n}$ be the $\sigma$-algebra generated by $e_{i}^{n}$ and define $\mathscr{G}^{n}=V_{i \in S} \mathscr{G}_{i}^{n}$. Moreover, we can assume that $\bigvee_{n \in \mathbb{N}} \mathscr{G}^{n} \subset \mathscr{G}$. Step 1 implies that for each $n \in \mathbb{N}$ there exists an allocation $\tilde{x}_{i}^{n}, i \in S$, which is feasible for coalition $S$, which is $\mathscr{G}^{n}$-measurable and for which

$$
\int u_{i}\left(\tilde{x}_{i}^{n}(\omega)\right) \mathrm{d} \mu(\omega) \geq \int u_{i}\left(x_{i}^{n}(\omega)\right) \mathrm{d} \mu(\omega)
$$

holds. Since $e_{i}^{n} \leq e_{i}$, it follows that each $\tilde{x}_{i}^{n}$ is an element of the order interval $\left[0, e_{S}\right]$ (recall that $\tilde{x}_{i}^{n}, i \in S$ is feasible for the coalition $S$ ). Since by Cartwright's Theorem the order interval $\left[0, e_{S}\right]$ is weakly compact, we can assume without loss of generality that each $\tilde{x}_{i}^{n}$ converges weakly to $\tilde{x}_{i}$. Since the set of all Bochner integrable and $\mathscr{G}$-measurable functions is weakly closed (recall Mazur's Theorem) it follows that each $\tilde{x}_{i}$ is $\mathscr{G}$-measurable. Moreover, the allocation $\tilde{x}_{i}, i \in S$ is feasible for coalition $S$. It now remains to take the limit on both sides of (5.6). Since $u$ is bounded and continuous, the expected utility is norm-continuous by virtue of the Lebesgue dominated convergence theorem. Thus, the right-hand side of (5.6) converges to the right-hand side of (5.3). Since the expected utility is weakly upper semi-continuous ${ }^{16}$ the lim sup of the left-hand side of (5.6) is less or equal to the left-hand side of (5.3). This proves Lemma 1.

It should be clear that in Step 1 the function $\tilde{x}_{i}$ is the conditional expectation of $x_{i}$ given $\mathscr{G}$ and that we use, in essence, Jensen's inequality for conditional expectations. The approximation argument in Step 2 can be used to show the existence of a conditional expectation for Bochner integrals and to extend a version of Jensen's inequality. This would provide an alternative proof of theorem 4 in chapter 5 of Diestel and Uhl (1977).

We are now ready to prove Theorem 2 .

Proof of Theorem 2. In order to prove the theorem we first show the following.

\footnotetext{
${ }^{15}$ A function $f$ is simple if and only if there exists a countable partition $A_{i}, i \in \mathbb{N}$ of $\Omega$ and $b_{i} \in Y$, $k \in \mathbb{N}$ such that $f=\sum_{i=1}^{\infty} b_{k} 1_{A_{k}}$ (recall that $Y$ is the Banach space).

${ }^{16}$ See Balder and Yannelis (1993, Theorem 2.8).
} 
Claim. Let $x_{i}, i \in S$ be a solution to

$$
\max _{x_{i}} \sum_{i \in S} \lambda_{i} \int u_{i}\left(x_{i}(\omega)\right) \mathrm{d} \mu(\omega),
$$

subject to

$$
\sum_{i \in S} x_{i}(\omega)=\sum_{i \in S} e_{i}(\omega), \quad \mu \text {-a.e. }
$$

Then there exists an alternative allocation $\tilde{x}_{i}, \in S$, which is feasible for coalition $S$, which is $\mathscr{G}$-measurable, and which gives the same expected utility to the agents as allocation $x^{i}, i \in S$.

In order to prove this claim, note that Lemma 1 implies the existence of an allocation $\tilde{x}_{i}, i \in S$, which is feasible for coalition $S$, which is $\mathscr{G}$-measurable and for which (5.3) holds. Since the original allocation $x_{i}, i \in S$ is a solution to (5.7), the equality must hold in (5.3) for all agents $i \in S$. This proves the claim.

The theorem now follows immediately from the claim. First note that each $\mathscr{G}$-measurable function is automatically $\mathrm{V}_{i \in S} \mathscr{F}_{i}$-measurable. This follows since by assumption (iv) in Section 2 each $e_{i}$ is $\mathscr{F}_{i}$-measurable. Hence, the information $\sigma$-algebras do not matter in the optimization problems. As a consequence, the induced TU game is independent of the information $\sigma$-algebras. Moreover, if $x_{i}$, $i \in I$ is a fine value allocation for $\mathscr{E}$ then $x_{i}, i \in I$ must solve (5.7) for $S=I$. Thus, we can use again the claim to replace $x_{i}, i \in I$ by a $\mathscr{G}$-measurable allocation $\tilde{x}_{i}, i \in I$, without affecting the agents' expected utilities. Thus, $\tilde{x}_{i}, i \in I$ is a fine value allocation. This concludes the proof of the theorem.

Theorem 2 demonstrates that the fine value allocation does not take into account the information superiority of an agent. Intuitively, this is the case because the only information that is relevant to a coalition $S$ is the endowment realizations of all members. However, this information is contained in $V_{i \in S} \mathscr{G}_{i}$ and hence in $V_{i \in S} \mathscr{F}_{i}$. As a consequence, the fine value allocation does not appear to be a useful concept for measuring informational asymmetries. This, together with the fact that the fine value allocation need not be coalitionally incentive-compatible (see Krasa and Yannelis, (1994)), makes this concept less attractive than the private value allocation that takes information asymmetries into account and which is also coalitionally incentive-compatible.

\subsection{The coarse value allocation}

The TU game $\left(V_{\lambda}^{c}, I\right)$ derived from a differential information economy need not be superadditive, ${ }^{17}$ i.e. there can exist coalitions $S$, $T$, with $S \cap T=\emptyset$ and $V_{\lambda}^{c}(S)+V_{\lambda}^{c}(T)>V_{\lambda}^{c}(S \cup T)$. On the one hand, this causes problems with the 
existence of a coarse value allocation. On the other hand, and (most importantly) this indicates that the coarse value allocation does not measure the marginal contributions of an agent to the coalitions of which he/she is a member. In particular, consider an agent, $i$, whose information is relatively coarse. Assume that $i$ joins a coalition $S$. Then trades within $S \cup\{i\}$ must be measurable with respect to the common knowledge information $\Lambda_{i \in S \cup[i]^{\mathscr{F}}}$. As a consequence, the trading opportunities of the members of $S$ decrease, and members of $S$ become worse off if agent $i$ joins. Thus, $V_{\lambda}^{c}(S \cup\{i\})-V_{\lambda}^{c}(S)<0$, which indicates that we measure the disutility agent $i$ imposes on members of $S$ rather than agent $i$ 's contribution to $S$. This is clearly not in the spirit of the Shapley value.

In order to illustrate this point, consider the following example.

Example 1. Consider an economy with three agents denoted by $I, J$ and $K$ and two states $\Omega=\{a, b\}$. Each state occurs with the same probability. There is one commodity in each state. All agents have the same von Neumann-Morgenstern utility function $u(x)=\sqrt{x}$. The endowments are given by $e^{I}=(9,0), e^{J}=(0,9)$ and $e^{K}=(0,0)$. Assume that $I$ and $J$ have full information, and that agent $K$ has only trivial information (i.e. $\mathscr{F}_{I}=\mathscr{F}_{J}=\{\{a\},\{b\}\}$, and $\mathscr{F}_{K}=\{\{a, b\}\}$ ).

In this economy, the coalition $\{I, J\}$ can achieve perfect risk-sharing. However, whenever agent $K$ joins, only state independent net-trades are possible. This leads to Shapley values of agents $I, J$ that are so high that they cannot be compensated any more by state-independent net-trades within the grand coalition. Similarly, the Shapley value of agent $K$ is negative for all weights $\lambda_{i}, i=I, J, K$.

In order to see this, note that the marginal contribution $V_{\lambda}^{c}(S \cup\{K\})-V_{\lambda}^{c}(S) \leq$ 0 for all coalitions $S$. Moreover, if $S=\{I, J\}$ the strict inequality holds, since the agents can trade before $K$ joins. However, in the grand coalition no trade is feasible. ${ }^{18}$ Since the Shapley value of agent $K$ is the weighted sum of these marginal contributions, we can conclude that $\mathrm{Sh}_{K}\left(V_{\lambda}^{c}\right)<0$. However, since expected utility is strictly positive, it therefore follows that there cannot exist an allocation $x_{i}, i \in I$ such that $\operatorname{Sh}_{i}\left(V_{\lambda}^{c}\right)=\lambda_{i} W_{i}\left(x_{i}\right)$, for all agents $i$.

In the above example, we choose agent $K$ 's endowment to be zero in order to simplify the presentation. The continuity of the Shapley value immediately implies that $\operatorname{Sh}_{K}\left(V_{\lambda}^{c}\right)<0$ also holds if we perturb the endowment slightly. Hence, the non-existence result is robust.

\footnotetext{
${ }^{17}$ Allen (1991) has made a related observation for the TU case that also applies to our framework. She has also proved existence results using a different argument than ours. Also, Myerson (1984) has proved existence results for the Nash bargaining solution with incomplete information. However, all of these results are of a different nature and do not cover our differential information economy framework.

${ }^{18}$ Because of the measurability restriction, all net-trades must not be state-contingent. Thus, unless the net-trades are zero, the consumption of some agents would become negative.
} 


\section{Acknowledgments}

We wish to thank two anonymous referees for helpful comments. As always we are responsible for any remaining errors. This research was supported by the Campus Research Board of the University of Illinois.

\section{References}

Aliprantis, C.D. and O. Burkinshaw, 1985, Positive operators (Academic Press; New York, London). Allen, B., 1991, Market games with asymmetric information: The value, Mimeo, University of Pennsylvania.

Balder, E. and N.C. Yannelis, 1993, On the continuity of expected utility, Economic Theory 3, 625-643.

Bewley, T., 1972, Existence of equilibria in economies with infinitely many commodities, Journal of Economic Theory 4, 541-540.

Cartwright, 1974, The order completeness of some spaces of vector valued functions, Bulletin of the Australian Mathematical Society 11, 57-61.

Dieste], J. and J.J. Uhl, 1977, Vector measures, Mathematical Surveys, 15 (American Mathematical Society, Providence, Rhode Island).

Emmons, D. and A.J. Scafuri, 1985, Value allocations-An exposition, in: C.D. Aliprantis et al., eds., Advances in Equilibrium Theory (Springer, Berlin, Heidelberg, New York).

Krasa, S. and N.C. Yannelis, 1994, The value allocation of an economy with differential information, Econometrica 62, 881-900.

Myerson, R.B., 1984, Cooperative games with incomplete information, International Journal of Game Theory 13, 69-96.

Shafer, W.J., 1980, On the existence and interpretation of value allocation, Econometrica 48, 467-474.

Shapley, L.A., 1953, A value of $n$-person games, in: H.W. Kuhn and A.W. Tucker, eds., Contributions to the Theory of Games, Vol. II (Princeton University Press, Princeton, NJ), 307-317.

Shapley, L.A., 1969, Utility comparisons and the theory of games, in: Guilbaud, ed., La Decision (Edition du CNRS, Paris, France).

Wilson, R., 1978, Information, efficiency, and the core of an economy, Econometrica 46, 807-816.

Yannelis, N.C., 1983, Existence and fairness of value allocation without convex preferences, Journal of Economic Theory 31, 282-292.

Yannelis, N.C., 1991, The core of an economy with differential information, Economic Theory 1, $183-198$. 